\title{
The Importance of a Visual Horizon for Distance Judgments under Severely Degraded Vision
}

\author{
Kristina M. Rand, Margaret R. Tarampi, Sarah H. Creem-Regehr, and William B. Thompson \\ University of Utah
}

\begin{abstract}
Two experiments examined the role of visual horizon information on absolute egocentric distance judgments to on-ground targets. Sedgwick (1983) suggested that the visual system may utilize the angle of declination from a horizontal line of sight to the target location (horizon-distance relation) to determine absolute distances on infinite ground surfaces. While studies have supported this hypothesis, less is known about the specific cues (vestibular, visual) used to determine horizontal line of sight. The current study investigated this question by requiring observers to judge distances under degraded vision given an unaltered or raised visual horizon. The results suggest that visible horizon information does influence perception of absolute distances as evident through two different action-based measures, walking or throwing without vision to previously viewed targets. Distances were judged as shorter in the presence of a raised visual horizon compared to the unaltered horizon. The results are discussed with respect to how the visual system accurately determines absolute distance to objects on a finite ground plane and for their implications for understanding space perception in low-vision individuals.
\end{abstract}

Perception of absolute distance is important for everyday navigational goals that rely on the ability to recover the scale of a space such as planning a locomotor path through an environment. Given that we perform tasks based on absolute scale with ease and accuracy over distances of several $10 \mathrm{~s}$ of meters, it is an intriguing problem to consider that there are only a few depth cues that can be used to recover absolute egocentric distances to locations more than 2-3m away from the observer. Sedgwick (1983) proposed what he called the horizon-distance relation as one mechanism by which a viewer standing on a ground surface might recover absolute egocentric distance to environmental locations also on that ground surface. The horizon-distance relation, often referred to as the angle of declination, suggests that the distance to a point on an infinite ground plane can be computed as a function of eye height and the angle between the line of sight to the horizon and line of sight to the object (see Figure 1).

In addition to describing the horizon-distance relation itself, Sedgwick raises two questions about how the visual system might utilize it to make distance judgments: (1) Is the visual horizon used to establish a frame of reference from which angle of declination to the target line of sight can be determined, or instead is the frame of reference established using proprioceptive information that relates to gravity-relative vertical? (2) If the visual horizon is in fact used, what happens with truncated ground planes, for which the visible horizon no longer corresponds to the "true" horizon corresponding to an infinite ground plane? The study reported on here provides evidence for the use of the horizon-distance relationship in human vision, and goes on to offer insights about the role of the visual horizon and the effects of a truncated ground plane.

Please address correspondence to: Kristina M. Rand, Department of Psychology, University of Utah, 380 S. 1530 E., Rm 502, Salt Lake City, UT 84112, kristina.rand@utah.edu. 
The horizon-distance relation builds on Gibson's (1950) ground theory of perception, which asserts that spaces are perceived within the context of support surfaces. While many potential surfaces exist, the most fundamental was suggested to be the terrestrial surface as it extends to the horizon, and the perception of such surfaces likely assists with judgments of depth, distance, size, etc. This is not entirely surprising provided that most action-based judgments involve perception of locations on the ground plane. Meng and Sedgwick (2001; 2002) demonstrated that even when objects were not in direct contact with the ground surface, the observer's perception of distance to the target was derived through mediated contact relationships, utilizing the point at which the intermediate surface made ground contact. This study, among others, provides support for the role of the ground plane in space perception.

Relatively few studies have been conducted to explore whether the horizon-distance relation is actually used in human perception. Of these, most have used prism goggle manipulations to alter the line of sight to target locations. For example, base-up prism goggles shift apparent lines of sight downward, causing an underestimation in distance judgments (Gardner \& Mon-Williams, 2001; Grutzmacher et al., 1997; Ooi et al. 2001; Thompson, Dilda, \& Creem-Regehr, 2007). However, base-up prisms shift the entire visual field downward, including not only the line of sight to the target, but also the line of sight to the visible horizon. As a result, the angle of declination between the line of sight to the target and the line of sight to the horizon remains unchanged. In fact, such prism goggle experiments are evaluating the effect of changing angle of declination with respect to gravity, not with respect to an actual horizon. In this paper, we explore whether or not distance judgments are also affected by manipulation of the angle of declination from the visual horizon to the target location. We also offer insights into the effect on distance judgments of the truncated horizon associated with finite-extent surfaces.

Research on visually perceived eye level has examined the relative contributions of gravitydefined and visually-defined information for judgments of eye level. In the absence of visual information, judgments of eye level are accurate, demonstrating the visual system's reliance on internally referenced gravitational information (Matin \& Fox, 1989). Given visual information for pitch of the ground plane discrepant with body-based cues, visually perceived eye level shifts, influenced by the environmental visual cues (e.g., Li \& Matin, 1995; Matin \& Li, 1995). While this is a distinctly different task than distance perception, the results point to the possibility of visual context affecting other perceptual judgments involving angle of regard.

Further support for the role of the horizon in distance perception comes from Ozban and Braunstein (2010). Using a desktop display, they showed that the vertical position of two objects relative to a visual indication of the horizon affected judgments of relative size, but not relative distance. They also showed that the effect was dependent on both the linear perspective vanishing point specified by converging lines and by the visual horizon itself, which in the case of a truncated ground plane is not colocated with such vanishing points. $\mathrm{Wu}, \mathrm{He}$, and Ooi (2007) showed that manipulations of patterns on the ground plane to change the apparent distance from the viewer to the vanishing point of the patterns affected absolute distance judgments, even when the visible horizon formed by the far termination of the patterns remained fixed. The work we describe below can be thought of as complementary to Wu et al. (2007), in that we explore the perceptual effect of changing the visible horizon, while holding other perspective information constant.

To date, only one experiment has explored the influence of manipulations to the visible horizon on perceived absolute distance. Messing and Durgin (2005) manipulated the visual horizon in a virtual outdoor environment. Motivated by a common finding of 
underestimation of distance judgments in virtual environments (i.e., Loomis and Knapp, 2003; Thompson et al. 2004), the visible horizon was lowered in an attempt to expand the environment, potentially correcting distance perception. Importantly, while absolute distance estimates, averaged over all distances did not differ between conditions, power function exponents of perceived distance as a function of intended distance were higher in the lowered horizon condition compared to the control. As the gravitational information available to participants was unaltered, the authors concluded that visible information may also contribute to determining horizontal line of sight. While this information is suggestive of the role of the visible information in the horizon-distance relation, it is critical to test this manipulation in a real-world setting.

A major obstacle that needs to be faced when exploring the role of the visible horizon on angle of declination in the real world is the methodology of the horizon manipulation. In the virtual environment scenarios such as that used by Messing and Durgin (2005), participants rely on an unfamiliar computer image to inform the horizon. In familiar real world settings, manipulations of the horizon are much easier to detect. Our goal in the present work was to draw on our previous methodology and findings using simulated low vision to make a horizon manipulation possible in a real world environment.

In Tarampi et al (2010) we found surprising results regarding absolute distance perception under simulated low vision. Participants walked without vision to a previously viewed target in both direct and indirect walking tasks after viewing the target through goggles with severely degraded contrast and acuity. Performance in the low vision simulation condition did not differ from that of a normal viewing condition. Thus, our results suggested that even without extensive experience with low vision, individuals are successful at utilizing available visual information to accurately detect absolute egocentric distances in the environment. Notably, the contrast between the back wall and floor in this specific environment was among the most salient, and may well have contributed to the success of distance judgments. Additionally, the degradation of contrast and acuity used in these procedures provides a methodological advantage to exploring the influence of the visible horizon, as alterations of the visible horizon are likely to go undetected.

We conducted two experiments exploring the role of the visible horizon on absolute distance judgments, which varied the way in which distance judgments were made. In both experiments, participants viewed on-ground targets, and were then asked either to walk without vision to the previously viewed target (Experiment 1), or to throw a beanbag to a previously viewed target (Experiment 2). All participants viewed targets under severely degraded vision. A horizon manipulation was made for half of the participants such that the floor/wall junction appeared to be higher than the unaltered horizon when viewed under low vision conditions. It was predicted that if participants are using visible horizon information to inform distance judgments, participants in the raised horizon condition would perceive objects to be closer than participants in the unaltered condition. Specifically, when the horizon is raised the angle of declination to the target increases, leading to underestimation in distance judgments. The alternative outcome of a lack of difference between the two conditions would lend support to an emphasis on the gravitational information when determining horizontal line of sight.

In addition to providing information about the use of visible horizon information for distance perception in indoor spaces, the study also has implications for low vision. While navigating through architectural spaces, low vision individuals likely rely on a limited set of visual cues for guidance. Understanding which visual cues are utilized is a critical component in understanding hazard detection and path planning. If visible horizon information does play a role in distance perception for low vision individuals, there are a 
number of implications for the design of space. For example, if a wall paneling does not have sufficient contrast to the adjacent floor, a false horizon similar to our manipulation may exist. While under normal vision that junction would be detectable, it may lead to misperceptions in distance to objects that may be potential trip hazards for low vision individuals.

\section{Experiment 1}

In the first experiment we used blind walking to previously viewed targets to assess distance judgments for individuals with simulated low vision. Blind walking is a visually directed action task that is well-established as an indication of perceived distance, and has been previously shown to reveal average accuracy in performance for targets presented out to at least 20 m (Fukusima et al. 1997; Loomis et al. 1992, 1998; Philbeck and Loomis, 1997; Rieser et al. 1990; Thomson, 1983). This procedure was used to examine whether manipulating the location of the visible horizon altered the perceived location of on-ground targets. As mentioned previously, if the horizontal line of sight used to determine angle of declination is derived, in part, from the visible frame of reference, participants viewing a raised visible horizon would be expected to underestimate the distance to targets compared to those in the unaltered horizon condition.

\section{Method}

\section{Participants}

A total of 38 individuals (19 males, 19 females, mean age 23.11 years) participated in Experiment 1. Participants were compensated with either partial course credit or were paid ten dollars. A total of eight participants were removed from analysis.

Of those removed, two participants were removed because they did not have normal vision, one participant was stopped so that they would not run into the back wall, and one participant was removed because they saw the second experimenter, therefore providing a size scale. An additional four participants were removed (two from each condition) because they failed to walk further to targets located at more distant locations.

\section{Materials}

To eliminate the distraction of environmental noises, all participants were equipped with noise-cancelling headphones. Participants listened to background pink noise on an mp3 player through the headphones while the experimenter spoke to the participant above the noise through a microphone. All participants also wore blur goggles that allowed for monocular viewing with their dominant eye, while the other eye was blacked out. The blur goggles were welding goggles with theatrical lighting gel (ROSCO Cinegel \#3047: Light Velvet Frost), resulting in an average acuity of $\log$ MAR 1.60, or 20/791 Snellen, fitted in the dominant eye. To block overhead and surrounding light cues, participants also wore a hood made of black fabric over the blur goggles. Three targets were used in the experiment, all of which were flat, isosceles trapezoids constructed of white foam core, providing a rectangular image on the retina when viewed from a prototypical eye height. To control for the visual angle of targets at varying distances, the targets were scaled to an appropriate size for the distance at which a participant viewed them. Dimensions (near width $\times$ far width $\times$ height) of targets placed at the egocentric distances of $3,4.5$ and 6 meters were $0.11 \times 0.12 \times 0.25 \mathrm{~m}$, $0.16 \times 0.17 \times 0.51 \mathrm{~m}$, and $0.20 \times 0.23 \times 0.90 \mathrm{~m}$, respectively. As it was imperative that participants did not have a view of the sidewall or ceiling as it would provide additional horizon cues, participants viewed targets from a position inside a viewing box. The viewing box was $0.91 \mathrm{~m} \times 1.2 \mathrm{~m} \times 2.1 \mathrm{~m}$, and was constructed with PVC piping and covered with black fabric. A foam-core viewing window attached via wooden hinges swung in from the 
side during viewing and allowed a 26-degree horizontal field of view (FOV). The only vertical restriction was the top of the viewing window, which eliminated the participants' view of the ceiling. Therefore the vertical field of view from the participants' feet to the back wall was unrestricted, and the FOV from the base of the back wall up was approximately 17 degrees. All participants were tested in a classroom with approximately $7.7 \mathrm{~m} \times 10.5 \mathrm{~m}$ of walkable space. On the back wall of the classroom, fabric was placed along the lower $0.65 \mathrm{~m}$ of the wall in the raised horizon condition. Participants' fixed starting point on all trials was positioned $9.83 \mathrm{~m}$ from the back wall of the classroom. For a participant of average eye height $(1.58 \mathrm{~m})$, the angle of declination from a horizontal line of sight to the actual floor/wall boundary was 9.1 degrees. In the raised horizon condition, the angle of declination to the apparent boundary was 5.4 degrees. Figure 2 shows images of the back wall of the classroom for both the raised and unaltered conditions, under normal and low vision.

\section{Design}

The experiment used a 2 (condition) $\times 3$ (distance) design in which condition (unaltered, raised horizon) was a between subject variable and distance $(3 \mathrm{~m}, 4.5 \mathrm{~m}, 6 \mathrm{~m})$ was a within subjects variable.

\section{Procedure}

After signing the consent form, participants' eye heights were measured and their eye dominance was tested. To familiarize participants with walking without vision, participants were first trained in the hallway outside of the classroom. The training procedure consisted of 1) experimenter-guided walking without vision, 2) independent blind walking in which the participant was instructed to start and stop walking without vision on command of the experimenter, and 3) two practice trials of blind walking to a previously viewed target without feedback. Participants were then presented with written instructions. After the training phase was complete, participants were instructed to put on the blur goggles and the hood, and were then led into the experiment room without prior vision of the interior of the classroom.

The experimenter led participants to the starting location inside the vision box. To allow participants to adjust to the task and to the vision box, the first four trials were treated as practice, but no feedback was given. Participants each performed 14 experiment trials; four trials each with targets at distances of 3, 4.5, and 6 meters, and two dummy trials to minimize memory strategies of target distances that could have occurred. The dummy trials occurred at 4 and 5 meters. A specific target was associated with each distance such that only one target type was presented at each distance. This allowed us to control for the visual angle of the targets. On each trial, the viewing window was swung in front of the participant prior to viewing. Participants were instructed to raise the hood and view the target. After viewing for 5 seconds, participants were instructed to lower the hood, close their eyes, and walk to where they believed the front of the target to be located. The viewing window was swung out of the walking path upon lowering of the hood so participants would run no risk of collision. The target was moved by an experimenter prior to contact. When participants stopped walking, the distance from the center of their final foot position to the starting location was measured. Participants were then guided along an indirect path back to the starting location to start the next trial. Upon completion of the task, participants were guided out of the classroom.

After the experiment trials, several vision tests were administered; the ETDRS Acuity test and the Pelli-Robson Contrast Sensitivity test. Participants were given each vision test twice, first while wearing the blur goggles, and a second time while wearing goggles allowing for 
clear monocular vision through the dominant eye. After the vision tests were completed, participants were debriefed.

\section{Results and Discussion}

A repeated-measures ANOVA was conducted on the absolute distances walked to each target distance, with Target Distance as a within-subjects factor and Horizon Group (unaltered/raised) as a between-subjects factor. We predicted that if participants were using the contrast of where the wall meets the floor to inform their visible horizon, that the Raised condition would underestimate target distances compared to those in the Unaltered condition.

Results for absolute distances walked by condition are shown in Figure 3. There was a main effect of Horizon Group [F $\left.(1,30)=4.24, \mathrm{p}<.05, \eta^{2}=.174\right]$, demonstrating that participants in the unaltered condition walked further than participants in the raised condition. The mean distances walked to targets $(3,4.5$, and 6 meters) were $3.13,4.59$, and 5.99 meters for the Unaltered group, and 2.86, 3.93, and 5.17 meters for the Raised group. There was also a significant main effect of Target Distance $\left[F(1,30)=259.11, p<.05, \eta^{2}=.926\right]$, indicating that participants walked further to targets that were further away. The Horizon Group by Target Distance interaction did not reach significance.

Experiment 1 demonstrated that perception of distance to on-ground targets, as indicated through blind walking performance, is influenced by manipulations to the visible horizon. That is, with otherwise identical viewing conditions, participants who viewed a raised horizon underestimated target distances when compared to participants who viewed an unaltered horizon. This direction of change in distance estimates between conditions is consistent with a hypothesis suggesting that visible information, at least in part, is considered when determining the horizontal line of sight for angle of declination information. When the visible horizon information was raised, and the line of sight to the target held constant, the angle of declination from the visible frame of reference was increased. As absolute distance and angle of declination have an inverse relationship, the apparent distance to targets decreased, likely leading to the observed underestimation. Also important to note about Experiment 1 results is how accurate participants were at walking to previously viewed targets in the unaltered horizon condition, despite simulated low vision. This result replicates the finding of Tarampi et al (2010).

\section{Experiment 2}

Experiment 1 used a blind walking procedure to investigate distance perception to onground targets under simulated low vision. Experiment 2 sought to replicate these findings using an alternative behavioral measure; blind throwing. Similar to blind walking, participants in a blind throwing task are asked view a target, lower a blindfold, and then throw a bean bag to the perceived location of the previously-viewed target. While not nearly as extensive as that for blind walking, past literature exists documenting blind throwing in both real and virtual environments (Eby \& Loomis, 1987; Sahm, Creem-Regehr, Thompson \& Willemson, 2005; Thomson, 1983)).

In addition to providing a secondary measure to increase confidence in the results of Experiment 1, blind throwing also has the benefit of providing a measure of distance estimates without egocentric spatial updating. In other words, while blind walking requires locomotion during task performance, blind throwing involves initiation of the task movement with no subsequent interaction with the environment or mental updating. Thus, these two tasks are distinct in the extent of dynamic updating of spatial location that they involve. The use of the throwing task allowed us to test whether the effect of the visible 
horizon found in Experiment 1 could be characterized as a perceptual effect that would generalize to a different measure or whether the effect was more specific to the visually directed locomotion task. In Experiment 2, the same alteration of the visible horizon was used as in Experiment 1 and participants underwent a very similar procedure, except instead of walking to the previously viewed targets, participants threw a beanbag to the target location.

\section{Method}

\section{Participants}

A total of 26 individuals ( 9 males, 17 females, mean age 22.6 years) participated in Experiment 2. Participants were compensated with either partial course credit or were paid ten dollars. A total of four participants were removed from analysis. Two participants were removed because they did not have normal vision, one due to throwing distances never exceeding $0.5 \mathrm{~m}$, and one for failure to follow task instructions. All remaining participants had normal or corrected-to-normal vision.

\section{Materials}

Many of the same materials from Experiment 1 were used in Experiment 2. Beanbags used in the throwing portion of the experiment were constructed from white cotton athletic socks and filled with birdseed. They were tied in a single knot at their base, with remaining fabric folded over the birdseed portion. The resulting beanbags, including sock and birdseed fill, each weighed $8 \mathrm{oz}$. For practice throwing, subjects threw beanbags to three targets on the floor with their eyes open. These targets were made of flat plastic material in varying shapes. The target distance during the experimental trials also differed from those used in Experiment 1. As such, different trapezoids were constructed from white foam core to control for visual angle. Dimensions (near width $\times$ far width $\times$ height) of targets placed at 3 , 4 and 5 meters were $0.11 \times 0.12 \times 0.25 \mathrm{~m}, 0.14 \times 0.15 \times 0.41 \mathrm{~m}$, and $0.17 \times 0.19 \times 0.62 \mathrm{~m}$, respectively. The manipulation of the horizon, viewing conditions, auditory conditions, FOV, and environment were identical to Experiment 1.

\section{Design}

The experiment used a 2 (condition) $\times 3$ (distance) design in which condition (unaltered, raised horizon) was a between subject variable and distance $(3 \mathrm{~m}, 4 \mathrm{~m}, 5 \mathrm{~m})$ was a within subjects variable.

\section{Procedure}

After signing the consent form, participants' eye heights were measured and their eye dominance was tested. Participants were then familiarized with the throwing task through pre-experimental training in the hallway. The first part of the training procedure consisted of eyes-open throwing to three on-ground targets placed at approximately $3.5 \mathrm{~m}, 4.5 \mathrm{~m}$, and 6 meters from the participant's starting location. Participants were instructed to throw the beanbags in an underhand motion to each target in succession, while keeping their feet stationary. The experimenter informed the participant which shape was to be thrown to on each trial. This process was repeated up to three additional times or until the participant demonstrated consistent throwing behavior. This process was designed to increase participants' confidence in accurate throwing as well as reduce variability during the experimental trials. Following the eyes-open training, blind throwing was introduced to familiarize participants to the experimental procedure. Three trials were administered, whereby participants were instructed which shape to throw to, allowed to lift the blindfold and view the target, lower the blindfold, and then throw the beanbag to the target location. 
No feedback was provided during this portion of the training. Participants were then presented with written instructions, equipped with noise cancelling headphones, low-vision goggles and hood, and led into the classroom. As in Experiment 1, no opportunity for a prior view of the classroom was provided.

Participants were led to the starting location inside the vision box. Instructions were repeated, followed by two practice trials. As before, 14 experimental trials were administered. Participants threw beanbags to each of the 3 critical distance, 3,4 , and $5 \mathrm{~m}$, three times each, in a random order. Additionally, two dummy trials were embedded at 3.5 and $4.5 \mathrm{~m}$. On each trial, the viewing window was swung in front of the participant prior to viewing. The experimental procedure was identical to Experiment 1 except that instead of walking to the previously viewed target, participants were instructed to throw the beanbag to the previously viewed target location on each trial. Careful attention was paid to the initial point at which the beanbag made contact with the ground, and distance was measured from this point to the participant's viewing location. Upon completion of the task, participants were guided out of the classroom. After the vision testing, also identical to those in Experiment 1, participants were debriefed.

\section{Results and Discussion}

A repeated-measures ANOVA was conducted on the absolute distances thrown to each target distance. Target Distance was analyzed as a within-subjects factor and Horizon Group (unaltered/raised) as a between-subjects factor. The same pattern of results as Experiment 1 was predicted in that the Raised condition would underestimate target distances compared to those in the Unaltered condition.

Results for absolute distances thrown by condition are shown in Figure 4. There was a main effect of Horizon Group $\left[\mathrm{F}(1,24)=7.40, \mathrm{p}<.05, \eta^{2}=.236\right]$, demonstrating that participants in the unaltered condition threw further than participants in the raised condition. The mean distances thrown to targets $(3,4$, and 5 meters) were $3.31,4.09$, and 5.03 meters for the Unaltered group, and 2.82, 3.50, and 4.10 meters for the Raised group. There was also a significant main effect of Target Distance $\left[F(1,24)=173.74, p<.05, \eta^{2}=.893\right]$, indicating that participants threw further to targets that were further away. The Horizon Group by Target Distance interaction did not reach significance. Also notable is the general accuracy seen in blind throwing to targets seen under severely degraded vision, further supporting the claim of Tarampi et al. (2010) that distance perception under these conditions is quite good.

Consistent with the findings of Experiment 1, the results in Experiment 2 demonstrate that alterations of visible horizon information influence the perceived distance to on-ground targets. Specifically, when the visible horizon was raised, participants underestimated target distances as indicated through their throwing behavior compared to participants presented with the unaltered horizon. These results are consistent with the predictions that participants were using angle of declination to inform distance judgments. This finding strengthens the argument that the visible frame of reference is considered when determining a horizontal line of sight by replication of Experiment 1 findings using a different behavioral measure.

\section{General Discussion}

We explored the role of visible horizon information on visually directed distance judgments in an indoor environment in two experiments. Of the few cues that provide information about the absolute scale of space, it has been suggested that the horizon- distance relation may be a mechanism that assists with such distance judgments. Specifically, viewers determine an absolute distance that is a function of the angle of declination from a horizontal 
line of sight and the line of sight to the target. To obtain the horizontal line of sight, observers may rely on visible information, gravitational information---either directly sensed through the vestibular system or indirectly determined through other proprioceptive senses, or a combination of both. While past studies have suggested that gravitational information is informative, our study aim was to determine whether or not participants utilize visible horizon information when making these judgments. In order to isolate visible horizon information without making the observers aware of the manipulation, we had participants view an unaltered or raised horizon environment under simulated severely degraded vision. We found that in both visually directed walking and throwing tasks, raising the visible horizon information led to an underestimation of absolute distance compared to an unaltered horizon. This finding is important both for the understanding of cues that contribute to distance perception in indoor spaces, and for implications of distance perception for a low vision population.

Our study is the first to empirically explore the effect on absolute distance perception due to direct manipulation to the visible horizon in a real world indoor environment. Thus, the study addresses two of Sedgwick's (1983) theoretical predictions about the use of angle of declination as a cue to recover absolute scale under normal viewing conditions, as stated in the Introduction. First, we show evidence that manipulating the visual horizon influences absolute distance judgments in the real world, addressing the question of whether the visual frame of reference is used. Second, we considered the case of an indoor environment in which the ground plane is truncated. If the visible horizon is used in such circumstances as if it was the horizon of an infinite ground plane, errors will be introduced into judgments of scale, and by implication, judgments of distance, with overestimation occurring for both (Sedgwick, 1983). In fact, while we find that the direction of the change in distance judgments as a function of the location of the apparent end of the floor is predicted by the horizon-distance relation, the actual magnitudes of the distance judgments are not (Figure 5). Instead, estimates for the unaltered condition are near veridical, while those for the raised floor-wall boundary show underestimation. Taken together with other results showing that both blind walking and blind throwing are accurate to target locations on an indoor, truncated ground plane, this is suggestive of a compensation strategy whereby observers are able to account for the truncated nature of their environments to preserve accurate egocentric distance perception to targets on the ground.

Results from the present study also have implications surrounding low-vision. The prevalence of blindness and low-vision in American adults over 40 years of age is a nontrivial 1 in 28, or a total of 3.3 million individuals (Congdon et al., 2004). Further, many individuals with low vision choose to navigate through environments using their remaining vision without external assistance (Ludt \& Goodrich, 2002). As they are likely able to utilize fewer cues to inform the layout of their surrounding space, it is important to investigate aspects that assist with or deter the safety of low vision navigation. The findings in this study suggest that architectural features that highlight visible horizon information may be important when recovering information about absolute distance to on- ground targets. For example, it may be important to highlight the contrast between walls and floors or the contrast between the floor and large features in open spaces or hotel lobbies. A potential direction for future research may be to focus on the point at which the horizon is raised beyond the point considered to be a believable false horizon. This information could contribute to guidelines on appropriate heights of architectural features that intersect the floor-wall boundary in order to prevent misperception of distance to potential hazards among low-vision individuals.

Importantly, the claims made about normal vision and reliance on the visible horizon for absolute distance perception need be taken cautiously. Our methodological goal was to 
manipulate the visible horizon under simulated low vision viewing conditions. As such, it remains possible that with improved acuity and contrast, individuals may have relied less on visible horizon information, reducing or eliminating the horizon manipulation effect. However, what remains important is that participants were able to extract an accurate scale of absolute distance from the limited information they were provided, and that this accuracy was compromised when the horizon information was altered. A potential direction for future research might be to explore ways of testing this same effect for normal viewing in real world environments or to generalize it further with virtual environment manipulations.

We also face a limitation in the ability to make general claims about low vision populations. All of the participants had normal or corrected-to-normal vision and viewed the environment under simulated low vision conditions. Therefore, it is highly unlikely that our sample has experience navigating throughout their environments with degraded contrast and acuity. The low-vision population, on the other hand, navigates throughout their environments daily without the visual abilities available to our participants. It is possible that through this experience, low vision individuals have learned to focus on other cues in their environment to help them recover absolute scale of space. However, some research also suggests that spatial competence of low vision individuals is not reliant on prior visual experience (see Loomis, Klatzky, Golledge, Cicinelli, Pellegrino \& Fry, 1993; Rieser, Hill, Taylor, Bradfield, \& Rosen, 1992). An important goal for future research would be to test the effect of horizon manipulations with a low vision population.

\section{Acknowledgments}

This research was supported by NIH Grant 1 R01 EY017835-01. The authors thank Benjamin Wheeler, Chuanjian Wang, and Klaryn Quirarte for their assistance in data collection, and Michael Geuss for his assistance with the construction of the vision box.

\section{References}

Congdon N, O’Colmain B, Klaver CC, Klein R, Munoz B, Friedman DS, et al. Causes and prevalence of visual impairment among adults in the United States. Arch Ophthalmol. 2004; 122(4):477-485. [PubMed: 15078664]

Eby DW, Loomis JM. A study of visually directed throwing in the presence of multiple distance cues. Perception \& Psychophyics. 1987; 41:308-312.

Fukusima SS, Loomis JM, Da Silva JA. Visual perception of egocentric distance as assessed by triangulation. Journal of Experimental Psychology: Human Perception and Performance. 1997; 23:86-100. [PubMed: 9090148]

Gardner PL, Mon-Williams M. Vertical gaze angle: absolute height-in-scene information for the programming of prehension. Experimental brain research. 2001; 136(3):379-385.

Gibson, JJ. The perception of the visual world. Boston: Houghton-Mifflin; 1950.

Grutzmacher, RP.; Andre, JT.; Owens, DA. Gaze inclination: A source of oculomotor information for distance perception. Studies in Perception and Action IV: Ninth International Conference on Perception and Action; New Jersey: Lawrence Erlbaum Associates; 1997. p. 229-232.

Li W, Matin L. Differences in influence between pitched-from-vertical lines and slanted- from-frontal horizontal lines on egocentric localization. Perception \& Psychophysics. 1995; 57:71-83. [PubMed: 7885809]

Loomis JM, Klatzky RL, Golledge RG, Cicinelli JG, Pellegrino JW, Fry PA. Nonvisual navigation by blind and sighted: assessment of path integration ability. Journal of Experimental Psychology: General. 1993; 122(1):73-91. [PubMed: 8440978]

Loomis, JM.; Knapp, J. Visual perception of egocentric distance in real and virtual environments. In: Hettinger, LJ.; Haas, MW., editors. Virtual and adaptive environments. Mahwah, NJ: Erlbaum; 2003. p. 21-46. 
Loomis JM, Silva JAD, Fujita N, Fukusima SS. Visual space perceptioin and visually directed action. Journal of Experimental Psychology: Human Perception and Performance. 1992; 18(4):906-921. [PubMed: 1431754]

Loomis JM, Klatsky RL, Philbeck JW, Gollege RG. Assessing auditory distance perception using perceptually directed action. Perception \& Psychophysics. 1998; 60:966-980. [PubMed: 9718956]

Ludt R, Goodrich GL. Change in visual perception detection distances for low vision travelers as a result of dynamic visual assessment and training. Journal of Visual Impairment \& Blindness. 2002; 96(1):7-21.

Matin L, Li W. Light and dark adaptation of visually perceived eye level controlled by visual pitch. Perception \& Psychophysics. 1995; 57:84-104. [PubMed: 7885810]

Meng JC, Sedgwick HA. Distance perception mediated through nested contact relations among surfaces. Perception \& Psychophysics. 2001; 63:1-15. [PubMed: 11304007]

Meng JC, Sedgwick HA. Distance perception across spatial discontinuities. Perception \& Psychophysics. 2002; 64:1-14. [PubMed: 11916293]

Messing R, Durgin F. Distance perception and the visual horizon in head- mounted displays. ACM Transactions on Applied Perception. 2005; 2:234-250.

Ooi TL, Wu B, He ZJ. Distance determination by the angular declination below the horizon. Nature. 2001; 141:197-200. [PubMed: 11700556]

Ozkan K, Braunstein ML. Background surface and horizon effects in the perception of relative size and distance. Visual Cognition. 2010; 18(2):229-254. [PubMed: 20814449]

Philbeck JW, Loomis JM. Comparison of two indicators of perceived egocentric distance under fullcue and reduced-cue conditions. Journal of Experimental Psychology. 1997; 23:72-85. [PubMed: 9090147]

Rieser JJ, Ashmead DH, Taylor CR, Youngqust GA. Visual perception and the guidance of locomotion without vision to previously seen targets. Perception. 1990; 19:675-689. [PubMed: 2103000]

Rieser JJ, Hill EW, Talor CR, Bradfield A, Rosen S. Visual experience, visual field size, and the development of nonvisual sensitivity to the spatial structure of outdoor neighborhoods explored by walking. Journal of Experimental Psychology. 1992; 121(2):210-221. [PubMed: 1534833]

Sahm CS, Creem-Regehr SH, Thompson WB, Willemsen P. Throwing versus walking as indicators of distance perception in real and virtual environments. ACM Transactions on Applied Perception. 2005; 1(3):35-45.

Sedgwick, HA. Environment-centered representation of spatial layout: Available visual information from texture and perspective. In: Beck, J.; Hope, B.; Rosenfeld, A., editors. Human and machine vision. New York: Academic Press; 1983. p. 425-458.

Tarampi MR, Creem-Regehr SH, Thompson WB. Intact spatial updating with severely degraded vision. Attention, Perception, \& Psychophysics. 2010; 71(1):23-27.

Thompson WB, Dilda V, Creem-Regehr SH. Absolute distance perception to locations off the ground plane. Perception. 2007; 36(11):1559-1571. [PubMed: 18265839]

Thompson WB, Willemsen P, Gooch AA, Creem-Regehr SH, Loomis JM, Beall AC. Does the quality of the computer graphics matter when judging distances in visually immersive environments? Presence: Teleoperators and Virtual Environments. 2004; 13(5):560-571.

Thomson JA. Is continuous visual monitoring necessary in visually guided locomotion? Journal of Experimental Psychology: Human Perception \& Performance. 1983; 9:427-443. [PubMed: 6223981]

Wu BW, He ZJ, Ooi TL. The linear perspective information in ground surface representation and distance judgment. Perception \& Psychophysics. 2007; 69(5):654-672. [PubMed: 17929690] 


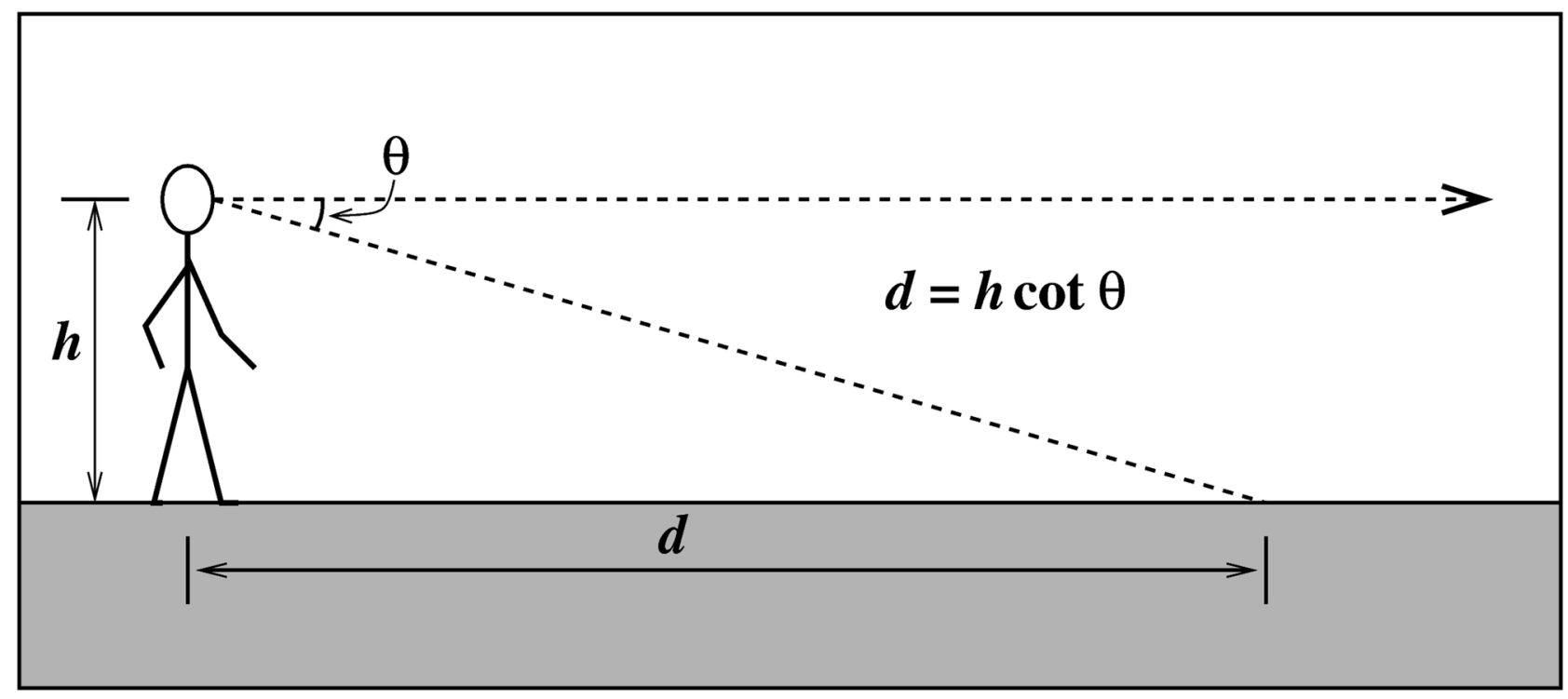

Figure 1.

Sedgwick's proposed calculation for egocentric distance provided angle of declination from horizontal $(\theta)$, and eye height $(h)$. 

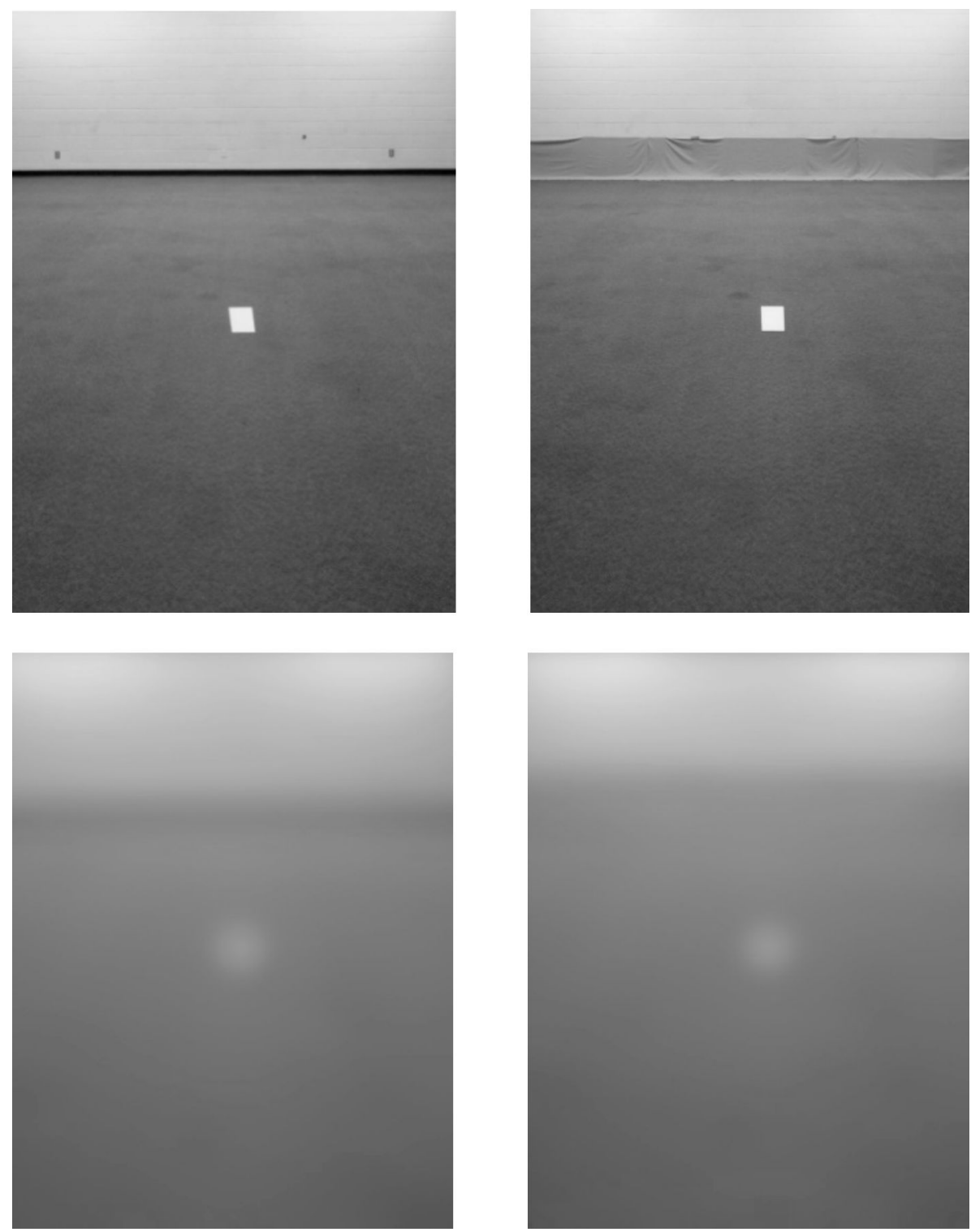

Figure 2.

Both unaltered (left) and raised (right) horizon experimental conditions through normal vision (top) and simulated low-vision (bottom). The top image was never shown to participants, but is demonstrated here to provide a view of the manipulation. 


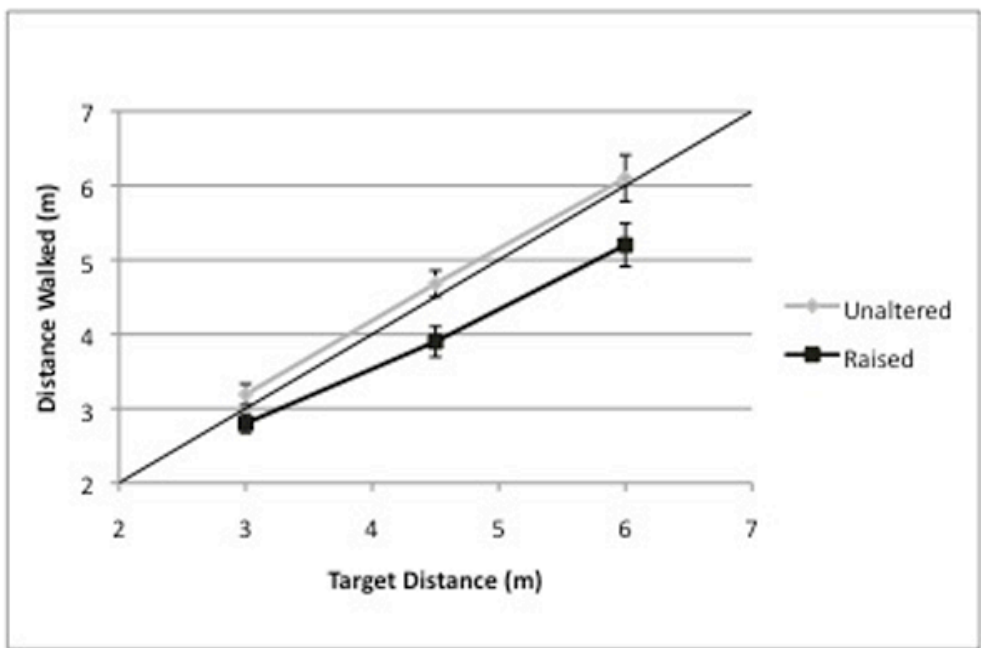

Figure 3.

Blind walking averages across subjects for 14 trials of blind walking to targets on the ground plane for Experiment 1. Error bars represent standard error. 


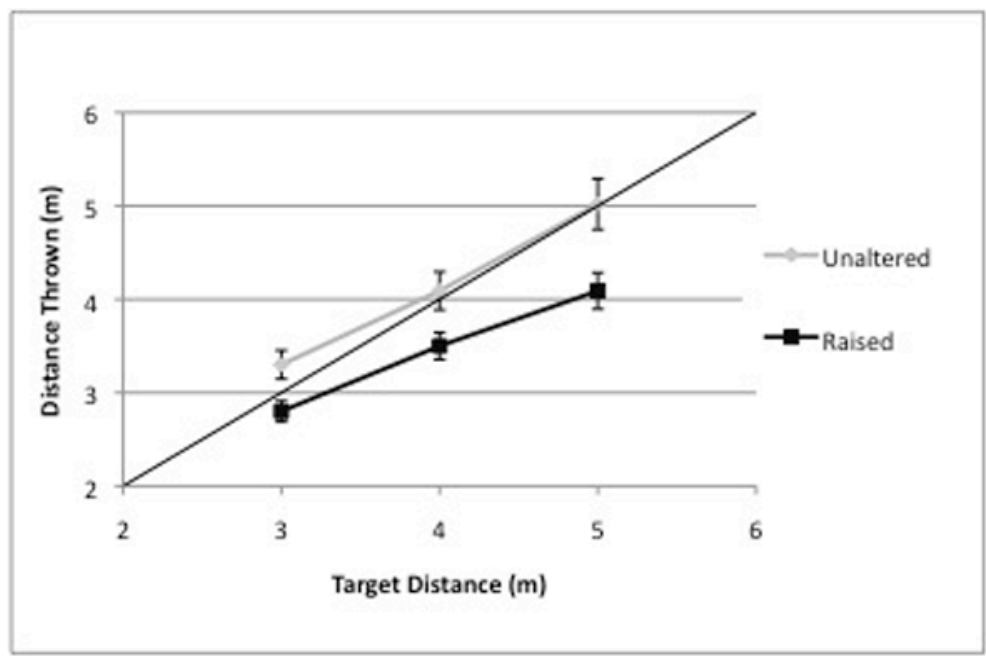

Figure 4.

Blind throwing averages across subjects for 14 trials of blind throwing to targets on the ground plane for Experiment 2. Error bars represent standard error. 


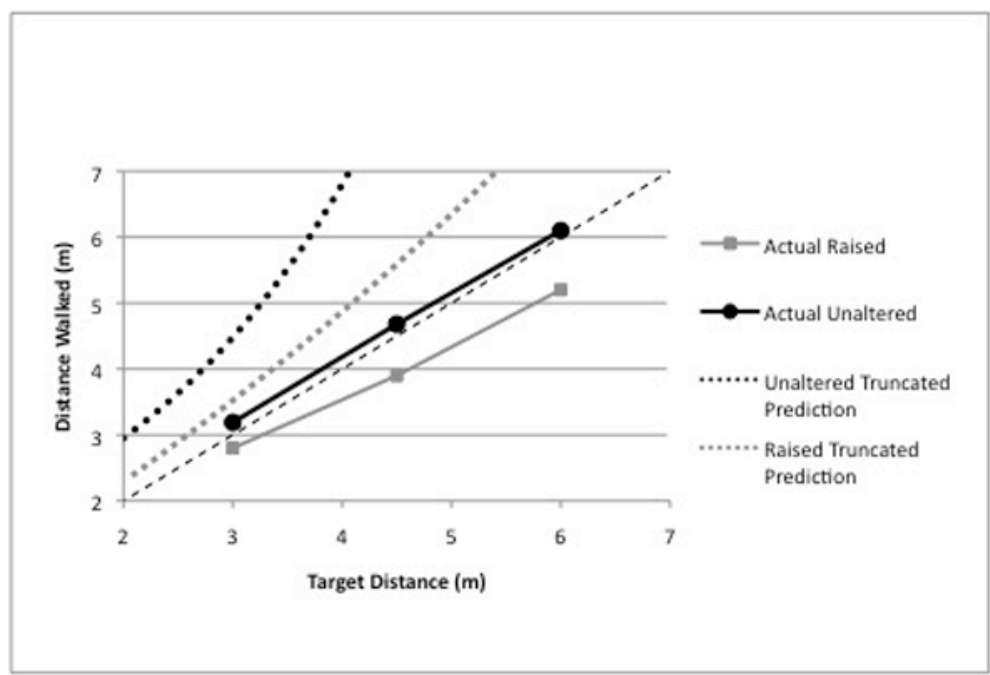

Figure 5.

Blind walking averages across subjects for 14 trials of blind walking to targets on the ground place for Experiment 1 as well as distance estimates as predicted by the horizon distance relation if floor/wall boundary acted as the true horizon. 